\title{
ANALYTIC MODEL OF DOUBLY COMMUTING CONTRACTIONS
}

\author{
T. BHATTACHARYYA, E. K. NARAYANAN AND JAYDEB SARKAR
}

Abstract. An $n$-tuple $(n \geqslant 2), T=\left(T_{1}, \ldots, T_{n}\right)$, of commuting bounded linear operators on a Hilbert space $\mathscr{H}$ is doubly commuting if $T_{i} T_{j}^{*}=T_{j}^{*} T_{i}$ for all $1 \leqslant i<j \leqslant n$. If in addition, each $T_{i} \in C_{.0}$, then we say that $T$ is a doubly commuting pure tuple. In this paper we prove that a doubly commuting pure tuple $T$ can be dilated to a tuple of shift operators on some suitable vector-valued Hardy space $H_{\mathscr{D}_{T^{*}}}^{2}\left(\mathbb{D}^{n}\right)$. As a consequence of the dilation theorem, we prove that there exists a closed subspace $\mathscr{S}_{T}$ of the form

$$
\mathscr{S}_{T}:=\sum_{i=1}^{n} \Phi_{T_{i}} H_{\mathscr{E}_{T_{i}}}^{2}\left(\mathbb{D}^{n}\right),
$$

such that $\mathscr{H} \cong \mathscr{S}_{T}^{\perp}$ and

$$
\left.\left(T_{1}, \ldots, T_{n}\right) \cong P_{\mathscr{S}_{\frac{1}{T}}}\left(M_{z_{1}}, \ldots, M_{z_{n}}\right)\right|_{\mathscr{S}_{T}^{\perp}}
$$

where $\left\{\mathscr{E}_{T_{i}}\right\}_{i=1}^{n}$ are Hilbert spaces and each $\Phi_{T_{i}} \in H_{\mathscr{B}\left(\mathscr{E}_{T_{i}}, \mathscr{D}_{T^{*}}\right)}^{\infty}\left(\mathbb{D}^{n}\right), 1 \leqslant i \leqslant n$ is either a one variable either a one variable inner function in $z_{i}$, or the zero function. 47B38

Mathematics subject classification (2010): 30H05, 46E22, 46M05, 46N99, 47A20, 47A45, 47B32,

Keywords and phrases: Hardy space over polydisc, doubly commuting contractions, shift operators, Sz.-Nagy and Foias model, isometric dilation.

\section{REFERENCES}

[1] P. R. AHERN AND D. N. CLARK, Invariant subspaces and analytic continuation in several variables, J. Math. Mech. 19 (1969/1970), 963-969.

[2] C.-G. Ambrozie, M. Englis And V. MüLler, Operator tuples and analytic models over general domains in $\mathbb{C}^{n}$, J. Operator Theory 47 (2002), no. 2, 287-302.

[3] N. Aronszajn, Theory of reproducing kernels, Trans. Amer. Math. Soc. 68 (1950), 337-404.

[4] W. ARveson, Subalgebras of $C^{*}$-algebras, III, Multivariable operator theory, Acta Math. 181 (1998), no. 2, 159-228.

[5] T. BhatTACHARYyA, J. ESCHMEIER, AND J. SARKAR, Characteristic function of a pure commuting contractive tuple, Integral Equations Operator Theory 53 (2005), no. 1, 23-32.

[6] R. CuRto And F.-H. VAsilescu, Standard operator models in the polydisc, Indiana Univ. Math. J. 42 (1993), no. 3, 791-810.

[7] S. W. DRURY, A generalization of von Neumann's inequality to the complex ball, Proc. Amer. Math. Soc., 68 (1978) 300-304.

[8] C. FefFerman And E. Stein, $H^{p}$ spaces of several variables, Acta Math. 129 (1972), no. 3-4, 137-193.

[9] V. Mandrekar, The validity of Beurling theorems in polydiscs, Proc. Amer. Math. Soc. 103 (1988), no. 1, 145-148.

[10] K. IZUCHI, T. NAKAZI AND M. SETO, Backward shift invariant subspaces in the bidisc II, J. Operator Theory 51 (2004), 361-376.

[11] V. MÜLlER AND F.-H. VASIlESCU, Standard models for some commuting multioperators, Proc. Amer. Math. Soc. 117 (1993), no. 4, 979-989. 
[12] G. POPESCU, Poisson transforms on some $C^{*}$-algebras generated by isometries, J. Funct. Anal. 161 (1999), no. 1,27-61.

[13] S. PотT, Standard models under polynomial positivity conditions, J. Operator Theory 41 (1999), no. 2, 365-389.

[14] W. Rudin, Function Theory in Polydiscs, Benjamin, New York 1969.

[15] J. SARKAR, Hilbert Module Approach to Multivariable Operator Theory, Handbook of Operator Theory, (2015) 969-1033, Springer Verlag, (edited by D. Alpay).

[16] J. SARKAR, Jordan Blocks of $H^{2}\left(\mathbb{D}^{n}\right)$, J. Operator Theory, 72 (2014), 371-385.

[17] J. SARKar, A. SASAne AND B. WICK, Doubly commuting submodules of the Hardy module over polydiscs, Studia Mathematica, 217 (2013), 179-192.

[18] B. Sz. NAGy And C. FoIAS, Harmonic Analysis of Operators on Hilbert Space, North Holland, Amsterdam, 1970. 